\title{
LA INNOVACIÒN CON TECNOLOGÍAS EN EL SISTEMA DE TRANSPORTE MASIVO E INTEGRADO URBANO BOGOTÁ - SOACHA Y SU IMPACTO EN EL DESARROLLO TERRITORIAL SUSTENTABLE
}

\author{
INNOVATION WITH TECHNOLOGY IN MASS TRANSPORTATION SYSTEM \\ AND INTEGRATED URBAN BOGOTA - SOACHA AND ITS IMPACT ON \\ SUSTAINABLE TERRITORIAL DEVELOPMENT
}

Daniel Molina Botache ${ }^{1}$

\section{RESUMEN}

En las siguientes líneas se pone a disposición el debate (inicial) sobre los impactos generados en el territorio por procesos de innovación y desarrollo tecnológico dentro del sector transporte terrestre urbano en su modalidad de pasajeros, más con la puesta en marcha de los sistemas masivos e integrados de transporte en el país, concretamente en el caso Bogotá D. C. y su vecino municipio de Soacha (Cundinamarca), en donde recientemente se han aplicado dichos sistemas repercutiendo en el grado de sustentabilidad del territorio y en otros aspectos como: movilidad e innovación de los sistemas de transporte integrados en diferentes sectores urbanos y regionales; el nuevo papel del Estado, de los Gobiernos y de la Mesa Sectorial del transporte en su función pública no sólo para controlar, inspeccionar y vigilar sino también en la generación de nuevas estrategias, programas y políticas incluso de privatización del sector; la intervención de la empresa privada en la operación de dicho sistema, la importación de autopartes y monopolio de combustibles; y hasta el propio ordenamiento territorial fijando nuevas infraestructuras, se han visto afectados y comprometidos desbordando los límites políticos -administrativos y exigiendo replantear la prospectiva territorial, teniendo en cuenta los actuales procesos de globalización, competitividad, innovación, productividad, medio ambiente y mejora en los tiempos reales de movilidad en regiones urbanas como el caso de la capital del país y sus periferias.

Palabras chaves: Territorio. Innovación. Transporte

\begin{abstract}
In the following lines are available to the (initial) discussion on the impacts on the territory by processes of innovation and technological development in the sector urban land transport in their mode of passengers, plus the launch of massive systems and integrated transport in the country, particularly in the case Bogotá DC and its neighboring municipality of Soacha (Cundinamarca), where they have recently implemented such systems impacting the degree of sustainability of the territory and in other aspects such as mobility and innovation integrated transport systems in different urban and regional areas; the new role of the state, governments and Transport Sector Board in its public function not only to monitor, inspect and monitor but

1 Universidad del Tolima - Colômbia. Endereço: 40, Cra. 74 \#42 C, Bogotá, Colômbia. Telefone: +57315 8400992. E-mail: danielmolina28@hotmail.com.
\end{abstract}


also in generating new strategies, programs and policies including privatization of the sector; the involvement of private enterprise in the operation of that system, import of auto parts and fuel monopoly; and to the territorial system itself setting new infrastructures, they have been affected and committed overflowing possible administrative political boundaries and demanding rethink the territorial prospective, taking into account the current processes of globalization, competitiveness, innovation, productivity, environment and improvement in real-time mobility in urban regions such as the case of the capital and its suburbs.

Keywords: Territory. Innovation. Transport.

\section{Marco Conceptual General}

En las siguientes líneas se propone adelantar más que una reflexión, un debate sobre los conceptos centrales de esta disertación, tales como: Transporte, Movilidad, tecnologías y territorios sustentables. Cuando se tratan los temas del Transporte y la Movilidad es necesario trazar las diferencias conceptuales propias, más si los hechos relacionados con ello son complejos y los tratamientos no sólo gubernamentales sino también de sociedades privadas como planificadores urbanos, ingenieros de diversa índole, entre otros, son reducidos y poco multidimensionales, por ello, sobre todo desde el siglo XX el desarrollo urbano está mediado por el Transporte Urbano, entendido como el "desplazamiento urbano" manifestado en (DANGOND-GIBSONE et al., 2013):
a) Personas: denominadas pasajeros.
b) Productos: recae en carga.

La precisión del transporte urbano se hace pertinente porque es en las ciudades donde se desarrollan las estructuras y sistemas tecnológicos motorizados que demandan nuevas miradas, políticas, estrategias, actores y dinámicas industriales y empresariales, el cual se clasifica en tres aspectos, a saber (Figura 1):

Figura 1 - Clasificación general del Transporte Urbano en Colombia 


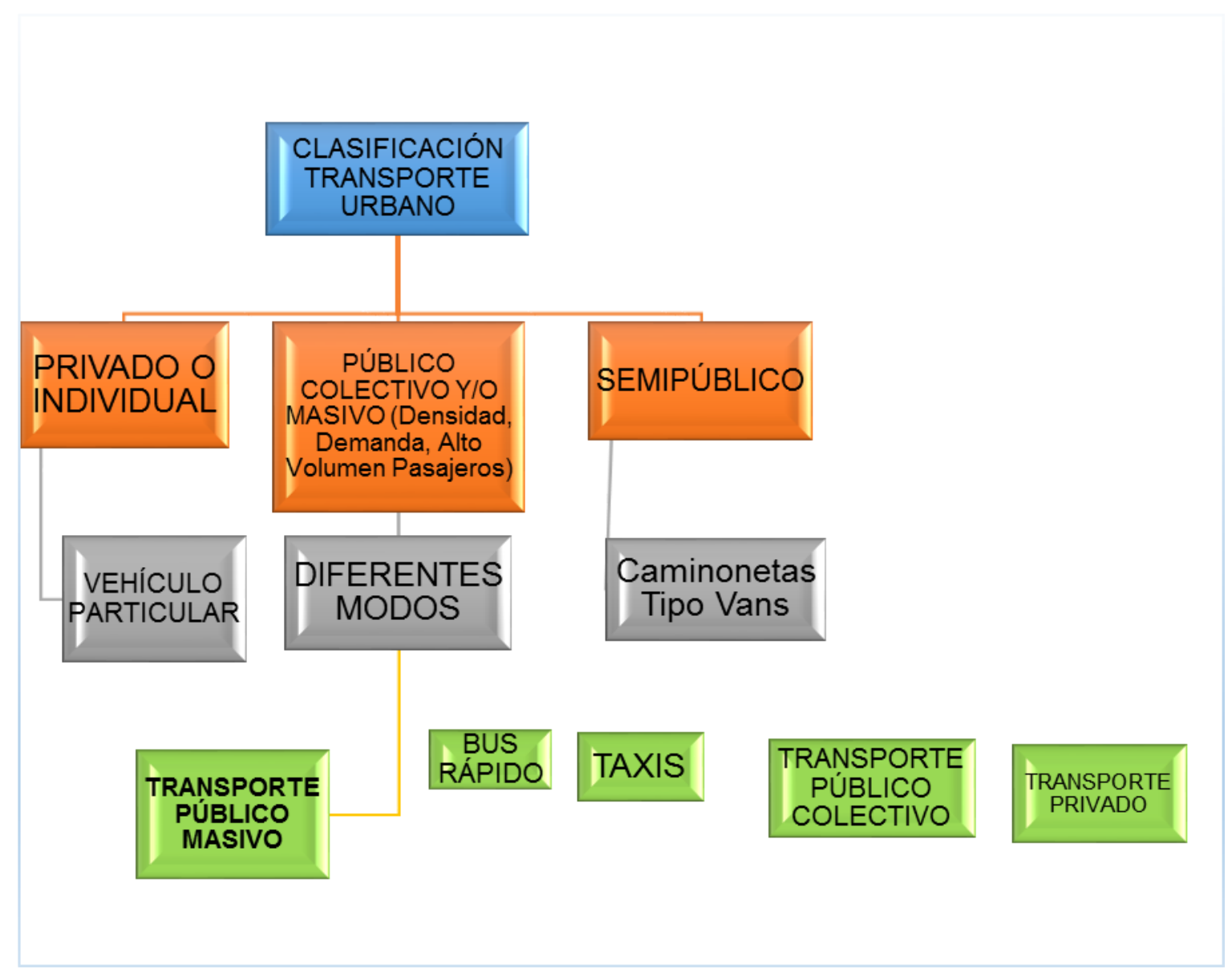

Fuente: El Autor, 2014.

De esta clasificación general se puede descifrar en los Diferentes Modos aspectos particulares, los cuales dinamizan al sector productivo, las redes de conocimiento, la oferta/demanda de mano de obra, diferentes actores, sociedad civil y al Estado. Entre estos aspectos se tiene:

a) Transporte Público: en muchos casos se cita los modos relacionados con el Metro y los Trenes (Riel) y Bus Rápido (BRT) por ejemplo en Colombia: buses del Sistema público Masivo como Transmilenio en Bogotá D. C., el Mío en Calí, entre otros; caracterizado por su alta capacidad de demanda de usuarios, vías exclusivas en doble sentido y articulados a los Sistemas Integrados de Transporte con Carriles preferenciales; tarifas, infraestructura y logística; estacionamientos y puntos de paradas fijas; uso de Sistemas de información para Usuarios a través de páginas Web y tarjetas electrònicas unificadas; redes sociales, chat, emails, mensajería instantánea; y apropiación de las nuevas tecnologías de cero o bajas emisiones como la electromovilidad en autos, motos y bicicletas, Chris, (2014) 
jalonada en el mundo urbano e industrializado por empresas de talla mundial con presencia en Latinoamérica; además de híbridos, hidrogeno y cable aéreo.

b) Transporte Público Colectivo: caracterizado por el transporte convencional de buses, colectivos, busetones y microbuses, cuyo sistema es operado por varias empresas y conductores propietarios de sus propios vehículos sin mucha capacidad logística, de infraestructura, capacitación profesional y tecnológica; junto a ello, la variedad de tarifas se extiende a la medida en la puesta en marcha de diferentes modelos de vehículos y rutas a los cuales la vida útil del vehículo se ha excedido y en temas de mantenimiento preventivo y correctivo presentan serias fallas, por ende generan mayor nivel de contaminación ambiental.

c) Transporte Privado: se enmarca en el uso -cada vez mayor- del vehículo particular y motocicletas en nuestro país, pero de manera centralizada, la cual la Asociación Nacional de Empresarios de Colombia, Andi, (2014) ha señalado la concentración del mercado automotor en cinco (5) ciudades del país principalmente (Tabla 1); mientras que el resto de ciudades del país representan ventas que no superan el $2 \%$ trimestral, lo que puede indicar un análisis de aspectos muy varios como lo es el uso del vehículo por marcas, dimensiones y volumen; tiempos de desplazamientos en diferentes trayectos, institucionalización del pico y placa en sectores o ciudades enteras por días a la semana; impacto ambiental en los territorios con la reducción de emisiones de gases contaminantes y ruido; y la necesidad de fomentar los Sistemas Masivos e Integrados de Transporte público. Junto a ello, la capacidad logística y administrativa para atender al sector en términos de mano de obra calificada en operación y mantenimiento especializado; el diseño de políticas públicas trazadas por diferentes actores como el Estado, gremios, academia, autopartistas, industrias y empresas importadoras, trabajadores de la industria como fabricantes y ensambladoras de vehículos y del sector transporte y sociedad civil; y finalmente la infraestructura física planeada y orientada por los Planes de Ordenamiento Territorial con componente ambiental. Factores sumados que ponen a la orden del día la complejidad de este tipo de transporte.

Tabla1- Acumulado ventas de Automóviles nuevos en ciudades de Colombia 2014 


\begin{tabular}{|c|c|c|c|}
\hline \multicolumn{5}{|c|}{ INFORME DEL SECTOR AUTOMOTOR -31 DIC/2014 } \\
TOTAL VENTAS 328.526 unidades \\
\hline \multicolumn{4}{|c|}{ ANDI } \\
\hline PUESTO & CIUDAD & $\begin{array}{c}\text { PORCENTAJE } \\
\text { TOTAL }\end{array}$ & $\begin{array}{c}\text { TOTAL } \\
\text { VEHÍCULO }\end{array}$ \\
\hline 1 & Bogotá D. C. & 36,8 & 65.986 \\
\hline 2 & Cali & 8.2 & 17.729 \\
\hline 3 & Envigado & 5.4 & 10.020 \\
\hline 4 & Medellín & 3.8 & 6.541 \\
\hline 5 & Barranquilla & 3.7 & 6477 \\
\hline 6 & Bucaramanga & 2.6 & 4.154 \\
\hline 14 & Cartagena & 1.0 & 1.546 \\
\hline
\end{tabular}

Fuente: ANDI, 2014

d) Taxis: de uso público no integrado al Sistema Masivo de Transporte Público en nuestro país, pero que es de gran importancia en las ciudades y municipios intermedios por su pertinencia y necesidad del servicio aun cuando el usuario no le sobra otra alternativa, la sobre oferta existente y concentrada en algunas empresas monopolistas, dinámica propia en la autorización de su puesta en marcha (valor económico del cupo para operación) por parte de las Secretarias de Movilidad y Transito; la mano de obra certificada y remunerada, y una serie de variables que lo hacen único, trascendental y de revisión especial (Tabla 2).

Tabla2 - Acumulado ventas de Taxis nuevos en ciudades de Colombia 2014

\begin{tabular}{|c|c|c|c|}
\hline \multicolumn{5}{|c|}{ INFORME DEL SECTOR AUTOMOTOR -31 DIC/2014 } \\
TOTAL VENTAS 328.526 unidades \\
\hline \multicolumn{5}{|c|}{ ANDI } \\
\hline \multirow{2}{*}{ PUESTO } & CIUDAD & $\begin{array}{c}\text { PORCENTAJE } \\
\text { TOTAL }\end{array}$ & TOTAL \\
VEHÍCULO
\end{tabular}


Fuente: ANDI, 2014

Por su parte el concepto "Movilidad" recoge al del Transporte y lo supera, en tal sentido se puede entender como el derecho a ir, a desplazarse a todas partes, tal definición se categoriza en la Constitución Política de Colombia como derecho fundamental en su artículo 24: "Derecho a circular libremente por el territorio nacional" (ALCALDIA MAYOR DE BOGOTÁ, 2014). Movilidad atañe al gran desarrollo urbano sobre todo generado por complejas y diversas dinámicas de finales del siglo XX e inicios del XXI, en la famosas megaciudades y urbes, teniendo en cuenta tres (3) aspectos marco, a saber:

1. Desplazamiento: de personas y mercancías, incluye acceso al servicio, calidad y satisfacción de necesidades, incluye

a) Infraestructuras: señalada por los espacios físicos apropiados para el diseño de vías; redes de telecomunicaciones, de orden institucional para atender, controlar, vigilar y mejorar el sector.

2. Vehículos: en este aspecto se pueden señalar la forma, tamaño, precios, autopartes, uso de tecnologías y gamas, mediado por la sostenibilidad ambiental, impactos sobre el territorio a la medida de la apropiación, modificación y cambio del paisaje y del espacio físico.

3. Gestión y organización de la Movilidad: aspecto trascendental de la unificación de los distintos modos en términos de seguridad, calidad de vida, atención al usuario-ciudadano y prestación del servicio; la protección del medio ambiente y junto a ello:

a) Dotación, diseño y construcción de equipamientos urbanos $\mathrm{y}$ tecnológicos a favor del sistema de transporte.

b) Solución de la movilidad para peatones, ciclistas, personas con movilidad reducida y no motorizados. 
c) Nueva cultura y roles de usuarios-ciudadanos y personas quienes realizan el desplazamiento de trayectos a través de los sistemas de transporte, el uso y cuidado de estaciones, entre otros casos.

d) Sistema jurídico favorable a la calidad del servicio y no al modelo rentista de quienes monopolizan la operación del sistema, también para establecer subsidios a grupos poblacionales.

e) Papel de los operadores a través de la fuerza laboral contratada en términos de salarios, horarios, capacitación y seguridad industrial.

f) Condiciones sociales, políticas y ambientales: relacionadas con la estructura social, poblaciones transportadas a centros de trabajo, comercio y de regreso a sus casas, otros aspectos con el fin de lograr y consolidar el desarrollo social, cultural y económico de las ciudades.

\section{Apuntes de la Movilidad Urbana Sostenible}

Sin duda alguna en el siglo XXI la movilidad es no sólo tema central de toda administración sino un problema multidimensional y complejo, que involucra, para su tratamiento, políticas del sector transporte y circulación, acceso y uso de espacios urbanos, priorización de modos no motorizados zonalmente $\mathrm{y}$ de tipo incluyente social $\mathrm{y}$ ambientalmente, por ejemplo: las tecnologías limpias; adicional capacitación profesional de trabajadores del sector, conciliación de política económica de fabricantes, ensambladores y concesionarios con el Estado y demás gremios, calidad del servicio para el usuarios y ciudadanía en general. En estos términos se hace necesario los enfoques mínimos en la Tabla 3, dicho escenario está priorizado por la movilización en diferentes modos alternativos de cero o bajas emisiones y sistemas de transporte público masivo e integrados (uso de la bicicleta en estacionamientos públicos y parqueaderos; ciclo rutas, ciclo vías permanentes, transporte motorizados híbridos de bajo impacto ambiental, etc), sin ningún tipo de determinismo económico, tecnocrático e ingenieril.

Para el caso concreto de la región de Soacha (Cundinamarca) y Bogotá D. C., como corredor vial principal del Sur de la Capital del país, el impacto negativo no sólo ambiental 
sino también territorial es gigante, al determinar ciertas variables como tiempos de traslado entre un trayecto a otro, por ejemplo de Soacha-San Mateo al Centro de la Capital, se ve afectado el tránsito vehicular por los grandes atascamientos de autos particulares, motocicletas, trasto-mulas, volquetas, carro-tanques, niñeras (vehículo pesado transportando vehículos livanos), ciclistas y transeúntes en medio de las vías por la falta de andenes y planificación del ordenamiento territorial, a grueso modo se puede hacer una lectura de ello a partir de la siguiente foto 1 . Si bien tras la puesta en marcha del sistema masivo de transporte público entre Bogotá D. C. y Soacha en el segundo semestre de 2014, éste no ha dado respuesta en conjunto a la movilidad y menos a la sostenibilidad ambiental territorial, en tal sentido en poco recorto tiempo de desplazamiento pero no es suficiente a la gran demanda del servicio por parte de trabajadores, usuarios y ciudadanos en general que requieren el traslado hacia el centro y norte de la urbe. 
Tabla 3 - Componentes de la Movilidad urbana Sostenible

\begin{tabular}{|c|c|c|}
\hline \multicolumn{3}{|c|}{ MOVILIDAD URBANA SOSTENIBLE } \\
\hline ASF & NECESARIOS & OOMPONENTES \\
\hline \multirow{5}{*}{1} & \multirow{5}{*}{$\begin{array}{l}\text { USODE } \\
\text { TEONOLOGIAS } \\
\text { LIMPIAS }\end{array}$} & $\begin{array}{l}\text { tecnologías } \\
\text { tradicionales }\end{array}$ \\
\hline & & Férreos \\
\hline & & Cable Aéreo \\
\hline & & Híbridos \\
\hline & & Eléctrico \\
\hline \multirow{3}{*}{2} & \multirow{3}{*}{ OULTURA } & $\begin{array}{l}\text { Conocimiento y } \\
\text { respeto de normas } \\
\text { sociales y juridicas }\end{array}$ \\
\hline & & $\begin{array}{l}\text { Uso adecuado y } \\
\text { mantenimiento de } \\
\text { veniculos ( Moto y } \\
\text { carro) prevención } \\
\text { yoperación. } \\
\text { (seouridad vial) }\end{array}$ \\
\hline & & $\begin{array}{c}\text { Calidad, Confianza } \\
\text { en elsistema, } \\
\text { costo del sistema. }\end{array}$ \\
\hline 3 & $\begin{array}{l}\text { ESPAOIOY } \\
\text { TERRITORIOS }\end{array}$ & $\begin{array}{c}\text { Dotación y } \\
\text { construcción de } \\
\text { paraues verdes, } \\
\text { recuperación de } \\
\text { zonas ambientales, } \\
\text { diseño de } \\
\text { infraestructuras, } \\
\text { carries exclusivos y } \\
\text { preferenciales, } \\
\text { componentes } \\
\text { socioambientales }\end{array}$ \\
\hline 4 & $\begin{array}{l}\text { ADMINISTRACION } \\
\text { YLOGISTIOA }\end{array}$ & $\begin{array}{c}\text { Financiero, } \\
\text { Administrativo y } \\
\text { Logistico, Mesas } \\
\text { Sectoraies, } \\
\text { Academ ía, } \\
\text { colombia (SENA - } \\
\text { Oentro de } \\
\text { Tecnologias del } \\
\text { Transporte -CTT-) }\end{array}$ \\
\hline 5 & SOCIEDAD CIVIL & $\begin{array}{c}\text { Empresarios, } \\
\text { sociedades de } \\
\text { autopartistas, } \\
\text { industrias, } \\
\text { oomuidades, } \\
\text { talleres. }\end{array}$ \\
\hline 6 & $\begin{array}{l}\text { OOMUNIDAD } \\
\text { INTERNACIONAL }\end{array}$ & $\begin{array}{c}\text { Fabricantes, } \\
\text { consorcios, } \\
\text { operadores } \\
\text { internacionales, } \\
\text { ejemplos exitosos }\end{array}$ \\
\hline 7 & ESTADO & $\begin{array}{c}\text { Politicas, Recursos. } \\
\text { Planes y Leyes. }\end{array}$ \\
\hline
\end{tabular}

Fuente: El Autor, 2014 
Foto 1 - Rasgos Soacha-Bogotá sobre Autopista Sur-Norte

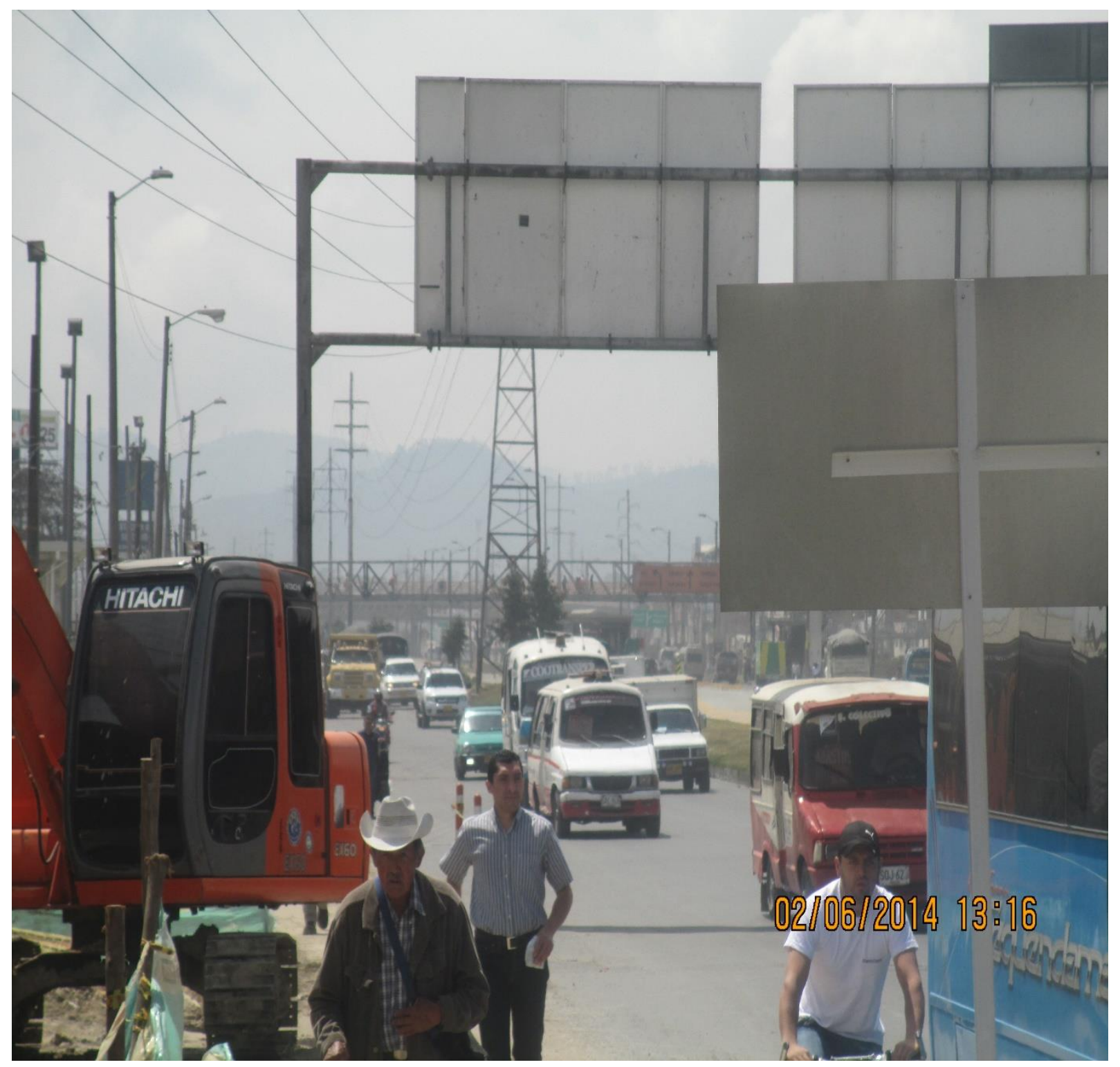

Fuente: El Autor, 2014

Sentido sur (al fondo de la foto) hacia el sentido de transitar al norte, trayecto hacia Bogotá D. C., un día hábil sin mayor congestión alrededor de las 13:00 horas, con algunas reparaciones sobre la vía de transeúntes, lo que vulnera la movilidad segura y ágil para estos autores de la vía, incluso para ciclistas y motorizados.

Foto 2 - Parque automotor vía Autopista Soacha- ingreso a Bogotá 


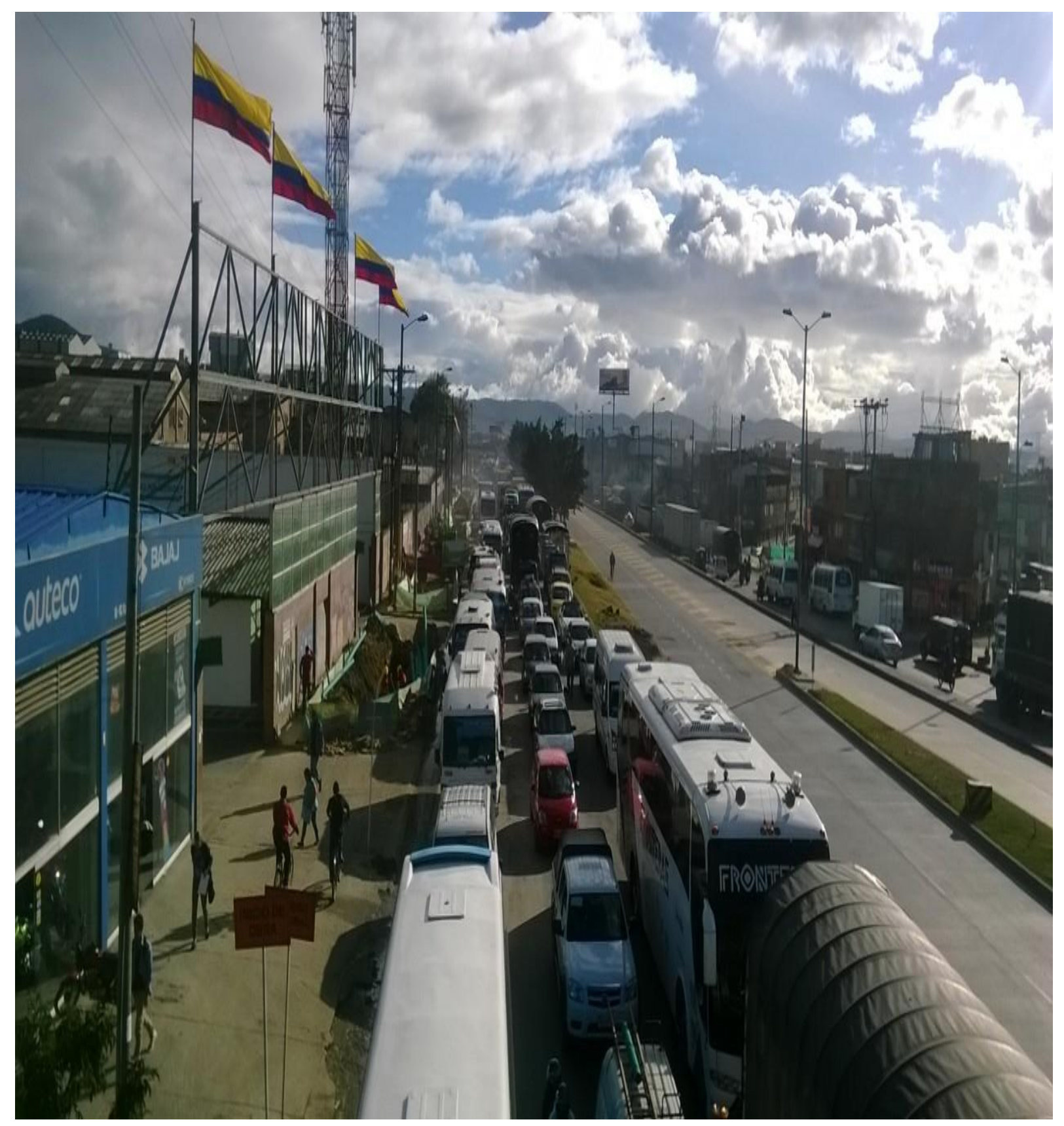

Fuente: El Autor, 2014.

En el sentido sur -norte (fondo de la foto) por la denominada autopista Sur (Bogotá Soacha) hacia las 13:00 horas, gran congestión vehicular en donde se pone en juego la planeación territorial ambiental, el uso de nuevas tecnologías en los sistemas de transporte público; el uso del vehículo particular, busetones intermunicipales y trascto-camiones en la vìa, junto a ello, peatones transitando al borde de las vías por los arreglos en los senderos 
peatonales. En el centro de la foto, se divisan los carriles exclusivos del sistema masivo de transporte público, Transmilenio (TM), como una alternativa no eficaz -por la alta demanda de usuarios- a la salida de los problemas de Movilidad en la ciudad capital de Colombia y sus municipios aledaños.

\section{Conclusiones}

1. Movilidad urbana sostenible trasciende el marco tradicional del transporte urbano público y privado, en dicho sentido involucra múltiples miradas, intereses, conveniencias, pero sobre todo: sostenibilidad ambiental, financiera, tecnológica y social a partir del uso de nuevas tecnologías del transporte público masivo e integrado, con el uso de transporte no motorizado en grandes conglomerados poblaciones incluso en transporte privado.

2. Los Sistemas de Transporte Masivo e Integrado van más allá de la aplicación de nuevas estrategias como el uso de restricciones al vehículo privado y de servicio público, se trata de un problema multidimensional de fabricantes, operación, mantenimiento, logística, el papel del Estado, trabajadores directos e informales, usuarios y ciudadanos y de los gobiernos de turno a través de políticas de Estado con trazabilidad financiera, social, ambiental-territorial y tecnológica en decenios.

3. En el ordenamiento del territorio los sistemas de transporte masivo e integrados enfocados en energías limpias cobra mayor vigencia a la par del desarrollo urbano del siglo $\mathrm{XXI}$, donde los factores económicos, sociales, ambientales y políticos son relevantes en las ciudades con más de quinientos mil (500.000) habitantes en el país, sobre todo, mientras en las ciudades y municipios con menos demanda del servicio de transporte masivo e integrado, tienen un papel importante los sistemas de transporte particular, en bicicleta, motocicletas los cuales también son objeto de análisis social, tecnológico y ambiental.

\section{Consideraciones}

a) Este es un debate inicial en torno a la movilidad urbana en especial a la implementación de nuevas tecnologías del transporte masivo e integrado en ciudades de Colombia, en especial Bogotá D. C. 
b) Lo que se expone y propone es un esbozo principal acerca de las mejorías en la movilidad, ordenamiento territorial, sistemas de transporte público, con una base social, ambiental y tecnológica sostenible.

\section{REFERENCIAS}

ANDI (Asociación de Empresario de Colombia). Informe sector automotor a julio 2014. Recuperado

$<$ http://www.andi.com.co/pages/proyectos paginas/proyectos detail.aspx?pro id=431\&Id=76 \&clase $=9 \&$ Tipo $=2>.2014$.

ALCALDIA MAYOR DE BOGOTA. Constitución Política de Colombia de 1991. Recuperado en: $\quad<$ http://www.alcaldiabogota.gov.co/sisjur/normas/Norma1.jsp?i=4125>. 2014.

CHRIS, L. La electromovilidad toma impulso. Con vehículos innovadores y conceptos inteligentes, Alemania desea ser el mercado de referencia. Recuperado en: $<$ https://www.deutschland.de/es/topic/economia/innovacion-tecnica/la-electromovilidadtoma-impulso>. 2014.

DANGOND-GIBSONE, et al. Del transporte a la Movilidad urbana en Bogotá. Más que un problema de vías y automotores. Editorial Universidad de Javeriana. Bogotá. 2013.

Recebimento dos originais: 27/08/2015

Aceitação para publicação: 08/11/2015 